\title{
Panel : Evolution and Changes in Information System Technologies in Industry
}

\author{
Panel Chair : \\ Sanya Uehara \\ Fujitsu Limited \\ 4-1-1 Kamiodanaka, Kawasaki, Kanagawa, Japan \\ s.uehara@jp.fujitsu.com
}

\section{PANEL DESCRIPTION}

Today, numerous standardization activities are going on around Web Services and XML, and they bring us evolution and changes in technologies for building distributed systems. For instance, they encourage loosely coupling integration of distributed systems with http-based protocols such as SOAP rather than remote procedure call based CORBA protocols. Also, they shift interface definition from API-based to XML-based, which makes it easier to extend interface among unknown parties. It is important to understand that how such standards change or improve the way to build distributed systems on the Internet with broad-band capabilities.

Software vendors are rapidly developing or extending many products to adopt the standards and associated technologies. The following is a list of such products:

- Network access servers: load balancing management, QoS management, security, access control

- Application platform: Web server, portal server, J2EE/EJB server, EAI/B2B server with workflow capability, UDDI 
-Application Development: web-front/portal, J2EE/EJB, process integration, data integration, etc.

However, the real business using SOAP or WSDL has not started yet in the real world. Most business is still within EAI, traditional B2B, or system enhancement, although some leading companies may try to evaluate such new standards. The business with CORBA based infrastructure is not increasing in the market of Japan, while webbased and mobile application is dramatically increasing. So, the software vendors are extending the products to incorporate advanced features based on such new standards, yet putting more energy to enhance the products to meet the traditional requirements from the customers.

So, the question is what kind of real benefits such new standards of technologies bring us, system developers, companies which run the system, or end-users. To answer that, we need to analyze the system which better uses such standards. Even a technologically sound standard will not be used unless it brings practical benefits to users. We need to discuss both how the standards may change the way we build the system, and also what benefits we will obtain from the standards.

Finally, we need to discuss the problems and future research areas keeping the above mentioned situations in mind. Such research areas may include (1)system development environment and tools, (2)software system architecture, (3)security issues, (4)highly reliable system that is realized by OS, middleware, and self-management capabilities. For each of these areas, we need to identify the problems and possible solutions. Apparently, the system development issues on the Internet today are the biggest ever, and plenty of research is needed in this area. 